Gefässchirurgie $2017 \cdot 22: 308$

DOI 10.1007/s00772-017-0281-8

Online publiziert: 13. Juni 2017

C) Springer Medizin Verlag GmbH 2017

CrossMark

M. Wortmann - D. Böckler · P. Geisbüsch

Klinik für Gefäßchirurgie und Endovaskuläre Chirurgie, Universitätsklinikum Heidelberg, Heidelberg, Deutschland

\title{
Erratum zu: Perioperative Liquordrainage zur Prävention der spinalen Ischämie bei endovaskulären Aorteneingriffen
}

Erratum zu:

Gefässchirurgie (2017)

DOI 10.1007/s00772-017-0248-9

Dieser Beitrag enthält eine fehlerhafte Literaturangabe auf S. 1. Bitte beachten Sie die nachfolgend korrekten Angaben:

\section{Inzidenz und Risikofaktoren für das Auftreten einer spinalen Ischämie}

Persistierende neurologische Ausfälle als Folge einer spinalen Ischämie im Rahmen der endovaskulären Therapie treten in etwa $2-8 \%$ der Fälle auf [5, 9-11, 32].

Der Verlag bittet, den Fehler zu entschuldigen.

\section{Korrespondenzadresse}

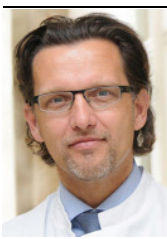

Prof. Dr. D. Böckler

Klinik für Gefäßchirurgie und Endovaskuläre Chirurgie, Universitätsklinikum Heidelberg Im Neuenheimer Feld 110, 69120 Heidelberg, Deutschland dittmar.boeckler@ med.uniheidelberg.de

\section{Literatur}

5. Patel HJ, Williams DM, Drews JD et al (2014) A 20-year experience with thoracic endovascular aortic repair. Ann Surg 260:691-696 (discussion 696-697)

9. Verhoeven EL, Katsargyris A, Bekkema F et al (2015) Editor's choice - ten-year experience with endovascular repair of thoracoabdominal aortic aneurysms: results from 166 consecutive patients. Eur JVasc Endovasc Surg 49:524-531

10. Wong CS, Healy D, Canning C et al (2012) A systematic review of spinal cord injury and cerebrospinal fluid drainage after thoracic aortic endografting. JVasc Surg 56:1438-1447

11. Greenberg RK, Lu Q, Roselli EE et al (2008) Contemporaryanalysis of descending thoracic and thoracoabdominal aneurysm repair: a comparison of endovascular and open techniques. Circulation 118:808-817

32. Jonker $\mathrm{FH}$, Trimarchi $\mathrm{S}$, Verhagen $\mathrm{HJ}$ et al (2010) Meta-analysis of open versus endovascular repair for ruptured descending thoracic aortic aneurysm. J Vasc Surg 51:1026-1032 (1032.e1021-1032.e1022) 